\title{
Study on properties of nano-epoxy resin based-low temperature plasma
}

\author{
Xudan Wang ${ }^{1, a}$ \\ ${ }^{1}$ Dalian University of Science and Technology
}

\begin{abstract}
In high-voltage power equipment, the traditional solid insulating dielectric is mainly polymer, epoxy resin (EP) is widely used because of its low price, superior performance, and easy processing. KH550 and low-temperature plasma fluorination in conjunction with KH550 modification in the paper is applied to improve nano-epoxy resin. EP samples with different treatment methods and different contents are prepared, and the insulation properties of EP materials before and after thermal aging are tested. It proves that the research results of the paper have a positive effect on the thermal aging characteristics of EP.
\end{abstract}

\section{Introduction}

Polymer surface fluorination technology can significantly improve the electrical properties of materials, and it is also the most used fluorination method in practice [1]. However, few people have studied the fluorination modification of nano-inorganic particles as fillers in nanocomposites. The effect of direct fluorination technology on nanofillers is remarkable. The plasma fluorination method is to use plasma technology to assist the introduction of $\mathrm{F}$ elements to the surface of the material, which should have the same effect on micro and nano inorganic particles. In response to this problem, according to published articles, the research team of Lu et al [2] has carried out research on it, and some results have been obtained. The research group analyzed plasma fluorination to improve the insulation performance of micro-AlN/EP materials. Obtained by SEM, XPS and FTIR, F element can be successfully grafted to the surface of AIN particles. It is found that after 45 minutes of fluorination, the particle size of AlN decreases by $26 \%$, and the content of $\mathrm{F}$ element is the largest, which can reach $38.55 \%$. And in the form of CF2 groups, this method can increase the charge dissipation rate of epoxy resin and increase the flashover voltage, and the optimal effect is $39.9 \%$ higher than that of pure EP [3].

Both the surface fluorination of high molecular polymers and the fluorination of micro and nano fillers can significantly improve the electrical properties of the material. This is attributed to the F element is the most electronegative element. The introduction of this element into the material can adsorb free electrons passing through the material and improve its insulation properties [4]. Therefore, Plasma fluorination technology in the paper is applied to assist the modification of nano-materials and prepare nano-epoxy composites. It is hoped to improve its electrical performance to enrich the comprehensive application performance improvement methods of polymer insulating materials, so as to meet the needs of high performance, low loss and extreme operating environments.

\section{Characterization and analysis of microscopic properties of modified nano-epoxy resin}

The surface morphology, element content, functional group and particle size of the material will change after modification by various physical and chemical methods, which in turn will change certain properties of the material, such as electrical, thermal conductivity and mechanical properties. Therefore, testing these changes is particularly important for material modification, which is an important way to prove the success of the method and whether it is good or not, and to analyze the reasons for the changes in material properties. The commonly used test methods include X-ray photoelectron spectroscopy (XPS), Fourier transform infrared spectroscopy (FT-IR), scanning electron microscope (SEM) and energy spectroscopy. In the experiment, these methods will be used to characterize the modification results [5].

\subsection{XPS analysis of modified nano-epoxy resin}

In the experiment, XPS is applied to test the surface elements of the modified epoxy resin powder. The XPS instrument parameters and experimental conditions used are shown in table 1 . 
Table 1 XPS instrument parameters and experimental conditions

\begin{tabular}{cc}
\hline Model & AXISULTRA, Kratos, UK \\
\hline Energy resolution & $<0.47 \mathrm{eV}$ \\
Spatial resolution & $<3 \mu \mathrm{m}$ \\
The smallest surface & $<15 \mu \mathrm{m}$ \\
Vacuum & $10^{-8} \mathrm{Torr}$ \\
Current & $10 \mathrm{~mA}$ \\
Voltage & $10 \mathrm{kV}$ \\
Background & Monochrome AlKa \\
\end{tabular}

The XPS spectra obtained after modification under
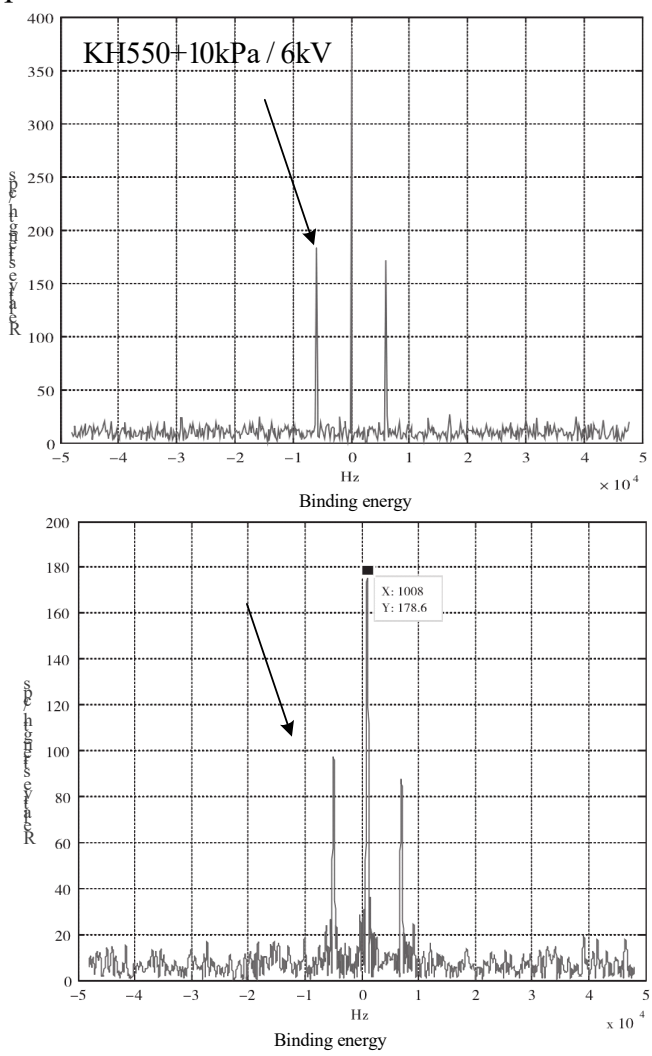

different conditions are shown in figure 1.

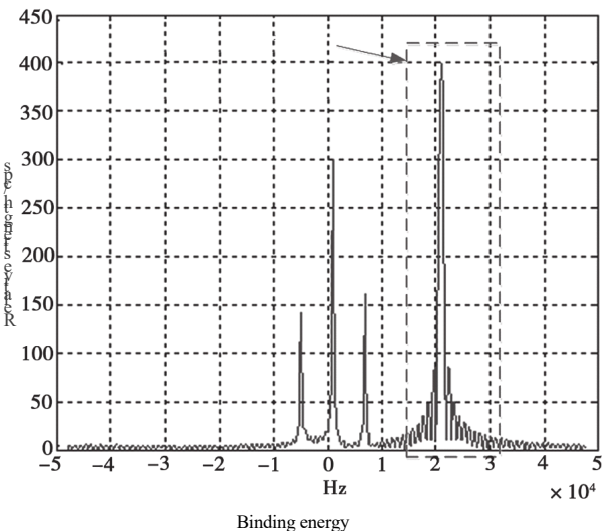

Figure 1 XPS spectrum under different modified conditions

$\mathrm{C}_{\mathrm{s}}(284.8 \mathrm{eV})$ is chosen for calibration. It can be seen from the figure that the $\mathrm{F} 1_{\mathrm{s}}$ peak appears in the XPS spectrum after plasma fluorination, and the F element is successfully introduced into the surface of the epoxy resin modified by KH550. It preliminarily shows that the method used in the experiment is feasible and has a certain effect, and with the increase of voltage and pressure, the peak value of element $F$ has increased significantly [6].

In order to further demonstrate the modification effect, the content of the surface elements of the epoxy resin is tested, and the results are shown in Table 2.

Table 2 Percentage of Epoxy resin surface element content after fluorination for 10 minutes

\begin{tabular}{cccccccc}
\hline \multirow{2}{*}{ Modification method } & \multicolumn{2}{c}{ Modification conditions } & \multicolumn{5}{c}{ Element mass percentage } \\
& Air pressure/kPa & Voltage/kV & $\mathrm{S}_{\mathrm{i}}(\%)$ & $\mathrm{O}(\%)$ & $\mathrm{C}(\%)$ & $\mathrm{N}(\%)$ & $\mathrm{F}(\%)$ \\
\hline \multirow{2}{*}{ KH550 } & 0 & 0 & 38.13 & 47.58 & 17.57 & 1.77 & 0 \\
& 10 & 7 & 37.72 & 38.89 & 18.07 & 0.97 & 8.72 \\
& 10 & 6 & 36.66 & 37.88 & 18.57 & 0.87 & 8.97 \\
Plasma fluorination & 13.5 & 5 & 35.78 & 37.24 & 19.06 & 0.86 & 8.08 \\
& 13.5 & 6 & 35.07 & 36.78 & 19.67 & 0.83 & 8.46 \\
& 13.5 & 7 & 35.01 & 36.06 & 19.93 & 0.42 & 10.05 \\
\hline
\end{tabular}


In Table 2, after plasma fluorination for 10 minutes, the $\mathrm{F}$ element reached about $8-10 \%$.

At the same time, since the $\mathrm{F}$ element is introduced into the surface of the epoxy resin, the content of $\mathrm{S}_{\mathrm{i}}, \mathrm{O}$, and $\mathrm{N}$ elements on the surface of the epoxy resin modified by KH550 will decrease [7]. However, the content of C element increased instead. This is because the main functional components in $\mathrm{CF}_{4} / \mathrm{N}_{2}$ plasma are $\mathrm{F}, \mathrm{CF}_{2}, \mathrm{CF}_{3}$, etc., and the $\mathrm{F}$ element introduced to the surface of the epoxy resin includes the introduction of F-containing methyl groups. Therefore, the content of $\mathrm{C}$ element will increase. Under the same modification time, when the air pressure is $13.5 \mathrm{kPa}$ and the voltage is $7 \mathrm{kV}$, the most $\mathrm{F}$ element is introduced, which is $10.05 \%$. Therefore, the air pressure is $13.5 \mathrm{kPa}$ and the voltage is $7 \mathrm{kV}$ [8].

\subsection{FT-IR analysis of modified nano-epoxy resin}

In order to characterize the changes of the epoxy resin surface functional groups after plasma fluorination, FT-IR analysis is performed on the epoxy resin modified by plasma fluorination $(13.5 \mathrm{kPa}, 7 \mathrm{kV})$ and $\mathrm{KH} 550$. The tablet method is applied. The dried $\mathrm{KBr}$ powder and nanoepoxy resin are mixed and grinded in proportion, then a small amount of $\mathrm{KBr} /$ epoxy resin mixture is taken to press tablets, and it is put into the instrument (FTIR-8900, SHIMADZU) for testing. The results of the KBr tablet as a background test are shown in Figure 2.

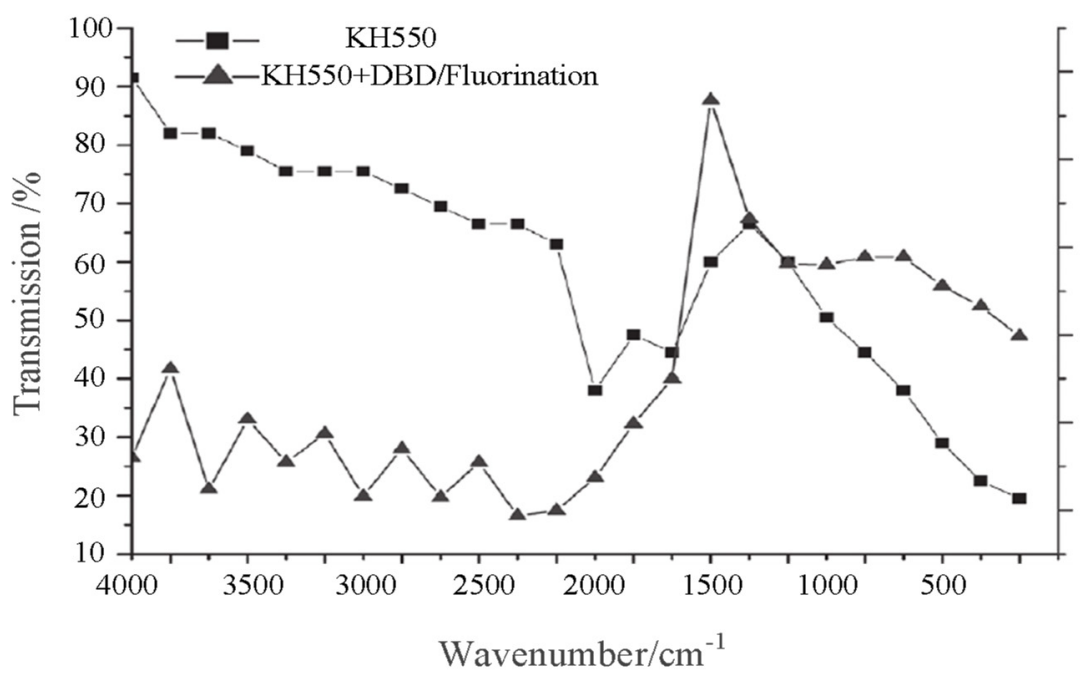

Figure 2 FT-IR analysis of epoxy resin after plasma fluorination

FT-IR only qualitatively analyzes the functional group changes on the epoxy resin surface after modification. According to the analysis in Figure 2, after KH550 modification, a new absorption peak appears on the surface of the nano-epoxy resin. Corresponding to - $\mathrm{CH} 3$ bending vibration peak is at $\lambda=1380 \mathrm{~cm}^{-1},-\mathrm{NH} 2$ absorption peak is at $\lambda=1643 \mathrm{~cm}^{-1}$, and $\mathrm{NH}_{2}^{+}$ absorption peak is in the range of $2430 \sim 2350 \mathrm{~cm}^{-1}$. $\lambda=1060 \mathrm{~cm}^{-1}$ represents the Si-O-Si characteristic peak in epoxy resin, and the absorption peak of $\lambda=799 \mathrm{~cm}^{-1}$ is the $\mathrm{Si}-\mathrm{O}$ symmetric stretching vibration peak. The $\mathrm{OH}$ absorption peak is in the range of $3400 \sim 3300$, which is the $-\mathrm{OH}$ contained in the surface of the nano-epoxy resin, it indicates that the KH550 modification is not completely coated on the surface of the epoxy resin [9]. Then the epoxy resin modified by KH550 is plasma fluorinated. It can be seen from the FT-IR chart that, in addition to all the above functional groups, only the $\mathrm{CF}_{2}$ absorption peak is at $\lambda=767 \mathrm{~cm}^{-1}$. It shows that after fluorination, the $\mathrm{F}$ introduced on the surface of the epoxy resin is mainly in the form of $\mathrm{CF}_{2}$, and the $\mathrm{F}$ in other forms is too small to be ignored. Moreover, although plasma has high energy, it can destroy the structure of KH550 grafted on epoxy resin when processing powder, but because the powder is spread on the dielectric plate, and the plasma generated by DBD is not complete Uniform, so the KH550 structure on the epoxy surface will not be completely destroyed. Therefore, the surface of the fluorinated powder still contains a large number of functional groups in KH550. From the above analysis, it can be seen that the epoxy resin treated by plasma fluorination modified KH550 is feasible [10].

\subsection{Energy spectrum analysis of modified nano- epoxy resin}

Using $7 \mathrm{kV}$ as the applied voltage, under the conditions of $10 \mathrm{kPa}$ and $13.5 \mathrm{kPa}$, the epoxy resin with different fluorination time is analyzed by energy spectrum. The results are shown in Figure 3. 


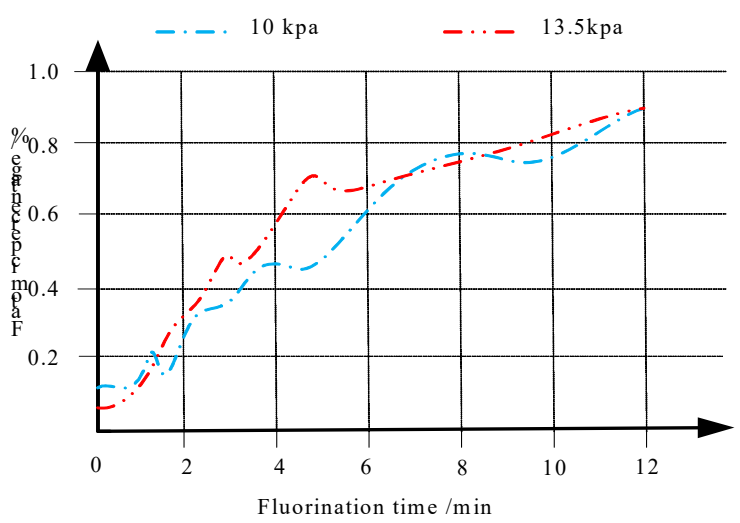

Fig.3 Energy Spectrum Analysis of epoxy resin with Different Time of Fluorination

In Figure 3, in the early stage of fluorination, the lower the pressure, the greater the power of the plasma, so in the

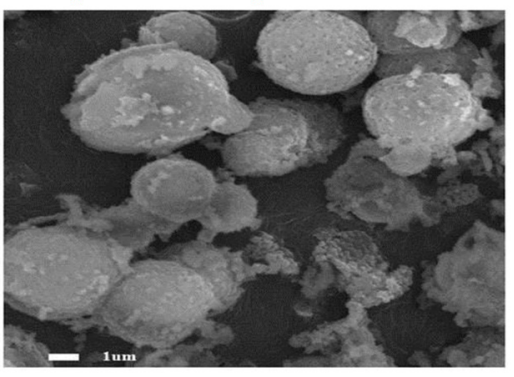

(a)Unfluorinated first 3 minutes, the content of $\mathrm{F}$ on the epoxy resin surface at $10 \mathrm{kPa}$ is more than that at $13.5 \mathrm{kPa}$. After 3 minutes, due to the decrease of the $\mathrm{F}$ content in the plasma, the fluorination effect at $13.5 \mathrm{kPa}$ is better than that at $10 \mathrm{kPa}$. Under the two pressure conditions, the $\mathrm{F}$ element on the surface of the epoxy resin grows slowly after 10 minutes. This is because the content of F element in the plasma is greatly reduced, which reduces the fluorination efficiency. Therefore, the fluorination time selected in this experiment is 10 minutes.

\subsection{SEM analysis of modified nano-epoxy resin}

The SEM test of epoxy resin powder before and after fluorination was carried out, and the results are shown in Figure 4.

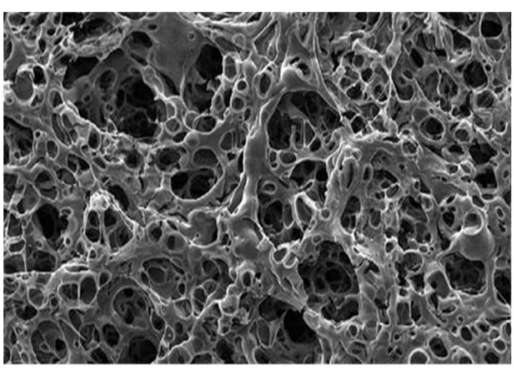

(b)Fluoride

Fig.4 SEM Image of Nano-silica

Observing the SEM images before and after the nanoepoxy resin powder treatment shows that the agglomeration problem of the epoxy resin powder after fluorination has been significantly improved. This is because during plasma fluorination treatment, the energy generated by gas ionization can destroy the van der Waals force and chemical bond between the epoxy resin particles, which can greatly reduce the agglomeration of the epoxy resin and make it better dispersed.

\section{Conclusion}

The paper introduces the platform and method of lowpressure plasma fluorination technology, and analyzes the feasibility and effect of the method in terms of plasma parameter control and microscopic. The conclusions are as follows:

(1) Based on the discharge characteristics and emission spectrum diagnostic technology, the discharge characteristics and distribution characteristics of CF4/N2 plasma are studied. The results show that the plasma distribution under the pressure and voltage conditions used is uniform, and the minimum energy generated is $0.497 \mathrm{eV}$, which can break the $\mathrm{C}-\mathrm{F}$ bond and provide conditions for the modification of epoxy resin.

(2) The analysis of XPS and FT-IR proves that the method is feasible. After fluorination for 10 minutes, the maximum content of $\mathrm{F}$ reached $10.05 \%$, and the element existed in the form of $\mathrm{CF} 2$, which can increase the content of $\mathrm{C}, \mathrm{F}$ and decrease the content of $\mathrm{Si}, \mathrm{O}, \mathrm{N}$, and this method could not achieve complete coating of epoxy resin.

(3) Using SEM and energy spectrum analysis, it is found that the best treatment time of low-pressure plasma fluorination is $10 \mathrm{~min}$. After fluorination for 10 minutes, the agglomeration problem of epoxy resin is significantly improved, and the dispersion is improved.

\section{References}

1. Duan S,Liu X,Wang Y,et al. plasma surface modification of materials and theirentrapment of water contaminant: A review[J].Plasma Processes and Polymers,2017,14(09):1600218.

2. Zhou Y,Li Q,He J L,et al.A scalable, high-throughput, and environmentally benignapproach to polymer dielectrics exhibiting significantly improved capacitiveperformance at high temperatures $[\mathrm{J}]$. Advanced Materials, 2018,30(49):1805672.

3. Zhang R B,Han Q T,Xia Y,et al.Plasma jet array treatment to improve thehydrophobicity of contaminated HTV silicone rubber[J].Plasma Science and Technology,2017,19(10):105505.

4. Preparation and performance of graphene waterbased anticorrosive spray paint $[\mathrm{J}]$. Lin Jieci, Chen Bingyao, Chen Mingyi, Wang Weisheng. Electroplating and Finishing. 2020(14)

5. Preparation and characterization of a functionalized graphene-based anticorrosive and heat dissipation coating[J]. Li Xiujuan, Wang Shoucheng, He Shengnan, Wang Xinchao, Yang Xuejing, Qian 
Dongwei. New Chemical Materials. 2020(06)

6. Preparation and characterization of large particle size graphite oxide and graphene [J]. Chang Ran, Pang Xiuyan, Li Zejiang, Xin Yaping. Journal of Hebei University (Natural Science Edition). 2020(03)

7. Research progress of graphene-based organic anticorrosive coatings[J]. Xiong Zhi. Guangzhou Chemical Industry. 2020(10)

8. Research progress of waterborne epoxy resin[J]. Zhang Ruizhu, Wang Chongyang. Henan Science and Technology. 2019(28)

9. Modification of graphene oxide and its application in waterborne epoxy resin [J]. Zhang Junfeng, Wu Lin, Liu Ying, Shu Shasha, Xu Jinhui, Liu Jiaming, Hu Ya. China Adhesives. 2019(02)

10. Talking about the preparation and performance of the new graphene/polypyrrole water-based anticorrosive coating $[\mathrm{J}]$. Zhang Yuxin. Chemical Management. 2018(05) 